\title{
Zur Darstellung der Bernoullischen Zahlen durch bestimmte Integrale.
}

\author{
Von Karl Carda in Wien.
}

Beziehungen zwischen Bernoullischen Zahlen und bestimmten Integralen wurden von mehreren Autoren aufgestellt. Die einfachste derselben ist wohl durch die Formel

$$
\left.\int_{0}^{\infty} \frac{x^{2 m-1} d x}{e^{2 \pi x}-1}=\frac{B_{m}}{4 m} 1\right)
$$

gegeben. Hierin bezeichnet, wie üblich, $B_{m}$ die $m^{\text {te }}$ Bernoullische Zahl, also

$$
B_{1}=\frac{1}{6}, B_{2}=\frac{1}{30}, B_{3}=\frac{1}{42}, \ldots
$$

Die Gleichung (1) lässt sich, wie nun gezeigt werden soll, auch auf folgendem instructiven Wege ableiten, der durch die einfache Bemerkung, dass die Function

die Transformation

$$
f(x)=e^{2 \pi x}
$$

$$
x \| x+i \quad i=\sqrt{-1}
$$

gestattet, nahegelegt wird.

Wir erstrecken das Integral

$$
\int \frac{(x+i y)^{2 m-1}}{e^{2 \pi(x+i y)}-1} d(x+i y)
$$

auf folgender geschlossenen Curve in der complexen Zahlenebene. Wir integrieren entlang der $x$-Axe von dem Punkte $(o, 0)$ zu dem Punkte $(a, 0)$. Dabei ist $0<\varrho<\frac{1}{2}$ und $a>\varrho$. Dann integriere

$\left.{ }^{1}\right)$ S a a I s ch ütz, Vorlesungen über die Bernoullischen Zahlen. Berlin 1893, p. 110. Monatsh. f. Mathematik u. Physilk. V. Jahrg. 
man parallel der $y$-Axe zu dem Punkte $(a, k)$, wobei $k$ eine ganze positive Zahl bedeutet, sodann von $(a, k)$ geradlinig nach $(a, k)$, dann auf einem Viertelkreise mit dem Radius $\varrho$ nach dem Punkte $(0, k-p)$, sodann geradlinig zu dem Punkte $(0, k-1+o)$, dann im ersten Quadranten auf einem Halbkreise mit dem Radius $e$ zu dem Punkte $(0, k-2+q)$ u. s. f., schließlich auf einem Viertelkreise von dern Punkte $(0, \varrho)$ zu dem Ausgangspunkte $(\varrho, 0)$. Die Summe dieser Integrale verschwindet. Das Integrationsgebiet wird durch die folgende Figur veranschaulicht.

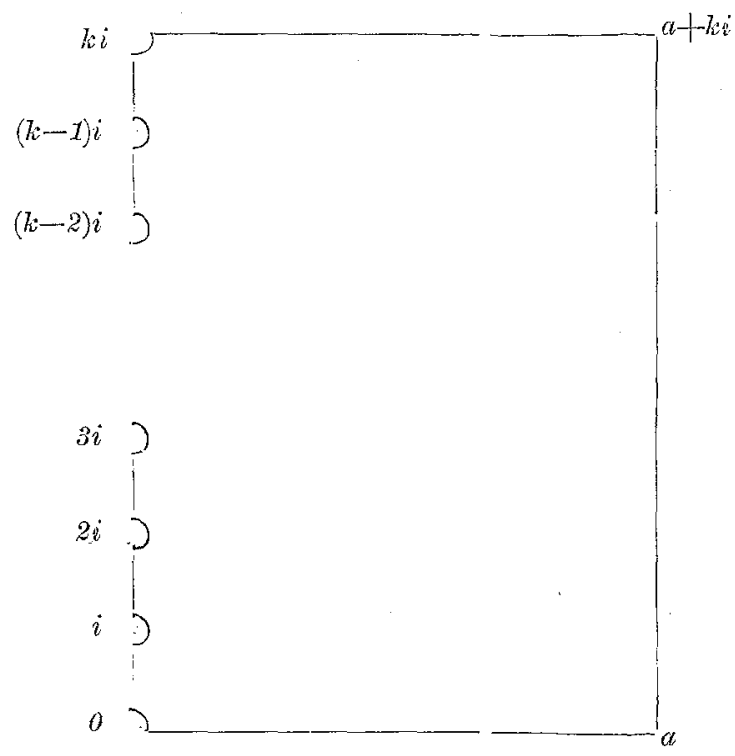

Dass die Radien der Halbkreise und der beiden Viertelkreise gleich gewählt wurden, ist offenbar keine Beschränkung. Für $\lim a=\infty$ verschwindet ein Term der Integralsumme und man erhält somit

$$
\left\{\begin{array}{c}
\int_{0}^{\infty} \frac{x^{2 m-1} d x}{e^{2 \pi x}-1}+\int_{\infty}^{e} \frac{(x+k i)^{2 m-1} d x}{e^{2 \pi x}-1}+\int_{((k i)))}+\sum_{\lambda=1}^{2=k-1} \int_{(\lambda i)}^{k}+ \\
+i \sum_{\lambda=1}^{\lambda=k} \int_{\lambda-e}^{\lambda-1+e} \frac{(i y)^{2 m-1} d \dot{y}}{e^{2 \pi i y}-1}+\int_{(j))}=0 .
\end{array}\right.
$$


Zur Darstellung der Bernoullischen Zahlen durch bestimmte Integrale. 323

Man hat nan in (2) ? gegen Null convergieren zu lassen. Man findet zunächst

(3)

$$
\begin{aligned}
& \lim _{\varrho=0} \int_{((0))}=0 ; \\
& \lim _{\varrho=0} \int_{((i i))}=\frac{(-1)^{m+1}}{n} \cdot k^{2 m-1} ; \\
& \lim _{\varrho=0} \sum_{\lambda=1}^{\lambda=1-1} \int_{\left(\lambda^{2} i\right)}=\frac{(-1)^{m+1}}{2} \cdot \sum_{\lambda=1}^{\lambda=k-1} \lambda^{2 m-1} .
\end{aligned}
$$

Ferner ist

$$
i \sum_{\lambda=1}^{\lambda=k} \int_{\lambda-\varrho}^{\lambda-1+\varrho}=(-1)^{m+1} \frac{i}{2} \sum_{\lambda=1}^{\lambda=1} \int_{\varrho}^{1-\varrho} \frac{(y-\lambda)^{2 m-1}}{\sin \pi y}(\cos \pi y-i \sin \pi y) d y .
$$

Durch Vergleichung der reellen Theile in (2) ergibt sich also für linı $\varrho=0$, wenn wir

$$
\int_{0}^{\infty} \frac{x^{2 \tau-1} d x}{e^{2 \pi x}-1}=T_{\tau}
$$

setzen,

$$
\sum_{\nu=1}^{\nu=m-1}\left(\begin{array}{c}
2 m-1 \\
2 \nu
\end{array}\right)(-1)^{\nu} k^{2 v} \cdot T_{m-\nu}+(-1)^{m}\left[\frac{k^{2 m-1}}{4}-\frac{k^{2 m}}{4 m}+\frac{1}{2} \sum_{\lambda=1}^{\lambda=k-1} \lambda^{\Sigma m-1}\right]=0 .
$$

Hieraus leitet man $a b$

$$
\sum_{\lambda=1}^{\lambda=k} \lambda^{2 m-1}=\frac{k^{2 m}}{2 m}+\frac{k^{2 m-1}}{2}+2 \sum_{\nu=1}^{\nu=m-1}\left(\begin{array}{c}
2 m-1 \\
2 \nu
\end{array}\right)(-1)^{m-\nu+1} k^{2 \nu} T_{m-\nu} .
$$

Setzt man $m+1$ an Stelle von $m$, so folgt
(5) $\sum_{\lambda=1}^{\lambda=k} \lambda^{2 m+1}=\frac{k^{2 m+2}}{2 m+2}+\frac{k^{2 m+1}}{2}+\sum_{\nu=1}^{\nu=m}\left(\begin{array}{c}2 m+1 \\ 2 \nu\end{array}\right)(-1)^{m-\nu} k^{2 \nu} .2 T_{m-\nu+1} \cdot$ 
Nun hat man aber die bekannte Formel

(6) $\left\{\begin{array}{c}\sum_{\lambda=1}^{\lambda=k} \lambda^{2 m+-1}=\frac{k^{2 m+2}}{2 m+2}+\frac{k^{2 m+1}}{2}+\sum_{\nu=1}^{\nu=m}\left(\begin{array}{c}2 m+-1 \\ 2 \nu\end{array}\right)(-1)^{m-\nu} k^{2 \nu} . \\ \left.\cdot \frac{B_{m-\nu}+1}{2(m-\nu}+1\right)\end{array}\right.$

Durch Vergleichung von (5) und (6) ergibt sich nach einfacher Überlegung

$$
\int_{0}^{\infty} \frac{x^{2 m-1} d x}{e^{2 \pi x}-1}=\frac{B_{m}}{4 m}
$$

$\mathrm{Zu}$ demselben Ergebnis gelangt man, wenn man die analoge Betrachtung auf das Integral

$$
\int \frac{(x+i y)^{2 m} d(x+i y)}{e^{2 \pi(x+i y)}-1}
$$

anwendet und dann die imaginären Theile vergleicht.

Schließlich sei noch bemerkt, dass Kronecker auf ahnlichem Wege die Gau $b^{\prime}$ schen Reihen summierte. ${ }^{1}$ )

1) Crelle's J., Bd. 105, p. 267. 